\title{
Animal models of wound repair: Are they cutting it?
}

\author{
David M. Ansell ${ }^{1}$, Kirsty A. Holden ${ }^{1,2}$ and Matthew J. Hardman ${ }^{1}$ \\ ${ }^{1}$ The Healing Foundation Centre, Faculty of Life Sciences, The University of Manchester, Manchester, UK; ${ }^{2}$ Epistem Ltd, Manchester, UK \\ Correspondence: Matthew J. Hardman, The Healing Foundation Centre, Faculty of Life Sciences, The University of Manchester, Manchester, UK, \\ Tel.: +44 161275 1578, Fax: +44 161275 3938, e-mail: matthew.j.hardman@manchester.ac.uk
}

Abstract: Current understanding of the complex process of wound repair is based on decades of study. Integral to this understanding has been the use of in vitro and in vivo models to uncover the key molecular players. Now that major wound processes are more fully understood, therapeutic strategies can be developed to manipulate wound repair. Particularly important areas for future research include developing therapies to aid treatment of healing pathologies such as chronic wounds, and manipulating the normal healing processes to drive a more regenerative phenotype in adults. Here, we discuss the benefits and limitations of current animal-based models and highlight the urgent need for improved predictive preclinical models for wound healing research. We conclude by suggesting directions where more robust models of chronic wound pathologies may arise, expediting the development of novel therapies.

Key words: chronic - in vivo - preclinical - translation - wound healing Accepted for publication 16 May 2012
Introduction - Why do we need models of healing? Wound repair is highly complex, comprising a series of coordinated and overlapping processes that have been characterised over many years For a review see $(1,2)$. The initial wound response, initiated immediately after injury, involves haemostasis with the resultant clot providing a scaffold and secreted factors to enable subsequent phases of healing. An inflammatory response removes local infection and debris, driving subsequent phases. A period of proliferation ensues to restore the epidermal barrier and to lay down granulation tissue and blood vessels. Finally, a remodelling phase results in partial restoration of functionality, yet this scar tissue confers at most $80 \%$ the strength of unwounded skin and is devoid of appendages. Tissue repair has been evolutionarily optimised for speed as opposed to perfect regeneration. There is currently much interest in harnessing latent reparative potential to promote regenerative healing in humans. Numerous models, both in vitro and in vivo, have been developed to study wound repair and identify key underlying mechanisms. In this article, we discuss whether currently available animal models are fit for purpose, particularly whether current models of pathological healing are sufficient for the identification of future therapies.

\section{Will in vitro systems ever 'replace' animal-based models?}

The 3Rs of animal experimentation (reduction, refinement and replacement) promote the development of alternatives to in vivo experimentation (3). As a community, we should strive to 'replace' sentient models (i.e. using live animals) wherever possible, or if animals are essential, to 'reduce' use to the absolute minimum. With respect to the study of wound repair, simple cell assays are available and widely used. These allow individual repair processes, such as proliferation or cell migration, to be investigated. However, the complex extracellular environment, paracrine and autocrine interactions and range of cell types involved in wound repair render these assays wholly inadequate as anything more than the most rudimentary of initial screens.
In response, more sophisticated in vitro organotypic culture models have been developed, albeit not directly for wound research. On the one hand, a reconstructed 3D "skin equivalent" can be produced (4). These generally use keratinocytes grown on a feeder layer of fibroblasts within a collagen gel, thus recreating rudimentary skin layers, which can be subsequently wounded $(5,6)$. Such a model can explore interaction between these two different cell types during wound repair, yet remains very simple, lacking important wound processes such as the inflammatory response or angiogenesis. On the other hand, a number of ex vivo skin explant systems have been developed (7). These models use whole excised skin, which should mirror the in vivo situation more closely, and contain all local cell types including some resident inflammatory cells. This technique has been applied to many model systems including pig (8), fish (9) and frog (R. Paus - Personal Communication), as well as from human skin obtained during cosmetic procedures (10). These have been injured in various ways such as UV damage (11), burn (12) and partial thickness excision $(13,14)$ to study repair processes and represent an important step towards meaningful non-in vivo models. However, these models remain particularly underdeveloped. Undoubtedly in the future, they will provide a viable non-sentient alternative for examining human repair processes and screening potential therapeutics. It seems unlikely that they will ever entirely recreate the in vivo wound environment, nor identify possible systemic or adverse effects of candidate therapeutic compounds. Moreover, ongoing difficulties in culturing skin explants long term mean they might ever only robustly model early phases of healing. Thus, although some initial experimentation can (and should) be performed in vitro, effective drug development currently requires in vivo models.

\section{If the pig is such a fantastic model, why don't more people use it?}

On paper, the pig would appear to be the ideal model for cutaneous wound healing studies. The structure of the skin is similar to that of a human, as opposed to the majority of other 'loose-skinned' 
animals. Furthermore, wounds heal in a similar manner to humans unlike rodents, where repair occurs more rapidly and predominantly via contraction (15). Yet, a simple search of PubMed listed articles since 2000 reveals only 193 publications under the terms 'wound healing in vivo pig', 1317 publications using 'wound healing in vivo mouse' and 439 with the terms 'wound healing in vivo rat'. It is clear therefore that despite the apparent departure from human repair, researchers prefer the rodent system.

It is likely that the apparent overlooking of the porcine model is mainly for logistical reasons, as many vivariums are not able to accommodate large animals. Moreover, when facilities are available, their large size and long gestation time ensure far greater financial and manpower costs compared with using rodents. Importantly, there are few transgenic pig lines available, with the first inducible line only very recently reported (16). By contrast, the wide availability of transgenic mouse strains allows for the disruption of individual genes to be tracked during healing. Indeed, a compendium of transgenic models displaying a wounding phenotype has been produced (17). Moreover, tissue-specific and/or inducible transgenic systems allow expression of a gene of interest to be restricted temporally and spatially during repair (18). With the caveat of known intrinsic differences to human repair, the mouse model system provides an abundant, manipulatable, accurate tool to study wound repair and is likely to remain the model of choice to mechanistically probe the process for the foreseeable future (19). Exactly how useful are current in vivo models? Animal models have been available for decades, yet historically in the wound healing field translation of candidates identified in animal models to human therapies has been problematic. Indeed, most will agree that while invaluable for uncovering important pathways and processes, current animal models are not well suited to preclinical evaluation of therapeutic candidates.

\section{What about regrowing limbs?}

With respect to the holy grail of scar-free healing, several in vivo models, such as zebrafish or xenopus embryos, display regenerative healing (20,21). Mammalian embryos also heal regeneratively until relatively late in gestation, studied extensively in sheep (22) and mice (23). Indeed, regenerative healing of human embryos was first described over 30 years ago (24), where scar-free healing occurs up to 24 weeks of gestation (25). Regeneration is not exclusively an embryonic phenomenon; adult salamander (Axolotl) and newt (Triturus cristatus) have been used as a regenerative model system for many years, exhibiting full limb regeneration (26). While limb regeneration has not been achieved in mammals, regenerative healing of mouse and human digit tip does occur (27). Moreover, the MRL mouse is a popular regenerative model where the ear heals regeneratively, while dorsal wounds heal with a scar (28). These models provide insight into how regeneration differs from reparation, with a long-term goal of reactivating human regeneration, rather than a vehicle for examining novel therapeutics per se.

There is currently a paucity of in vitro regeneration models that can be used to bridge the gap between initial high-throughput screens and the subsequent more in-depth analysis of promising leads. Recently, an ex vivo model using axolotl skin has been described (29), although more work is required in this area.

\section{What do I want to show?}

It is first important to stop and consider why you are contemplating wounding in the first place. Do you want to: (i) mechanisti- cally understand normal healing; (ii) mechanistically understand pathological healing or; (iii) explore a potential new wound treatment? The choice of model ultimately rests with the answer to this question, as the optimal model will depend on the exact aims of study. Unfortunately, experimental design is far too often based on 'our lab uses this model', with perhaps a more favourable alternative being overlooked.

What follows is a summary of the choices currently available. How should I make my wound?

Full-thickness surgical excisions and full-thickness incisions, performed on mouse dorsal trunk skin, are by far the most popular wound models. That said, numerous variations in experimental design are employed by different groups. Excisional wounds, for example, can be (and are) made in many different shapes and sizes, either by biopsy punch, surgical scissors or laser/burn. Wounds can be left open (termed healing by secondary intention) or splinted, sutured or dressed. Excisional wounds dressed with Tegaderm are suggested to aid assessment of wound reepithelialisation (30), while secondary intention involves greater wound contraction (31). Tegaderm dressings are of particular note because they create a 'moist' wound environment, which alters many aspects of subsequent healing (32). Incisional wounds are often sutured to resemble the clinical situation bringing periwound tissue into close proximity. Here, wound strength becomes a clinically useful measure (33), and this has been suggested as a particularly reproducible model for scarring research (34). Compounds can be delivered locally to the wound via topical application and peri-wound injection or incorporated in dressings, or administered systemically.

The optimal wounding protocol (e.g. type, size, dressings or sutures) will differ depending on the exact purpose of the study, yet there remains little consensus within the field. As a result, comparison between different publications is often difficult. An important goal for the future should be standardisation of the wounding protocol and subsequent data analysis. Such a 'refinement' would maximise the possibility of corroborating studies.

While the vast majority of wounds are made on mouse trunk (dorsal) skin, healing progression will vary by species and body site. This should be taken into account when selecting a more exotic wound model. It should be noted, however, that a less mainstream model may actually prove more effective depending on the context, for example, the rabbit ear for evaluating hypertrophic scars (35). The merits of different species for wounding studies are outlined in Table S1 (online), while a recent review highlighting several more obscure models is also available (36).

The popularity of excisional and incisional models lies in the fact that wounding can be performed quickly and reproducibly in any strain. Moreover, multiple validated outputs can be employed to assess healing and uncover mechanisms. The most basic analysis involves daily photographs to profile macroscopic wound closure, although more complex methods will yield additional information. Histological analysis is frequently employed allowing researchers to measure wound depth in addition to width, and crucially to assess proliferation, inflammation and other wound processes. These models have greatly advanced our understanding of wound repair. Especially important has been the extensive use of transgenic models to examine the role of wound processes over time (37), and the function of specific genes in different cell types 
during repair (38), which would be impossible in vitro. Now that normal healing is beginning to be understood, an important goal for the future is to identify strategies to improve repair in pathological conditions such as venous leg ulcers, diabetic foot ulcers and pressure ulcers, where healing is delayed.

\section{What if I want something more complicated?}

In humans, defects in normal healing lead to pathological fibrosis or non-healing chronic wounds Fig. 1. Chronic wounds (diabetic foot ulcers, venous ulcers and pressure sores), which cost healthcare providers over $£ 3$ billion per annum in the UK alone (39), develop through different underlying pathology, yet display remarkable similarities. Current treatments are far from optimal, with therapy centred on treating secondary symptoms (40). The lay public is often shocked to hear that standard care of chronic ulcers still includes use of maggot therapy, a treatment more associated with the First World War battlefield than 21st century medicine. The development of drug-based therapies for chronic wounds should be of utmost importance given the increasing incidence of ageing- and obesity-related pathologies.

So you have identified an exciting entirely novel process in normal mouse wound healing, which may or may not be important for pathological healing. What next?

\section{Diabetic models}

The most widely used models of impaired healing involve diabetic mice or rats. Models of type 1 diabetes exist such as genetically deficient non-obese diabetic (NOD) mice (41), or via chemical ablation of pancreatic beta cells through streptozotocin (STZ) injection (42). While these models also display a delayed wound repair phenotype, these may not represent the ideal model to study diabetic foot ulcers, which are prevalent in type 2 diabetes (43).

The most widely used models of type 2 diabetes are leptin (ob/ $\mathrm{ob})$ - or leptin receptor $(\mathrm{db} / \mathrm{db})$-deficient mice $(44,45)$. These mice become obese from around 6 weeks of age and subsequently develop type 2 diabetes, with a pronounced delay in wound repair $(46,47)$. However, it has been postulated that in this model, the impaired healing phenotype may be more related to the leptin pathway knockout than hyperglycaemia (48), related to differences in mechanical properties of the skin, or simply reflect the sheer obesity of the animals (49). Several polygenic diabetic strains have more recently been developed (50-52) with wound repair defects. Type 2 diabetes may also be modelled by substituting standard chow for high-fat diet (53), although this is not routinely used for wound healing. Confusingly, the observed wound repair phenotype differs between the diabetic models described and wound type employed $(48,54,55)$, complicating predictivity of the human clinical state. The diabetic mouse model has been successfully used to identify the therapeutic potential of numerous growth factors (56-59), yet despite this, recombinant human PDGFbb (Becaplermin gel; Regranex) is the only drug to have successfully completed human trials and achieved FDA approval to treat diabetic foot ulcers (http://www.fda.gov/Drugs/DrugSafety/PostmarketDrugSafety InformationforPatientsandProviders/DrugSafetyInformationfor HeathcareProfessionals/ucm072121.htm. accessed 6th Jun 2012). Repeated use of Regranex has been associated with increased cancer risk highlighting the need for additional drug-based therapies and preclinical wound models. The fact that only a singledrug therapy has emerged, despite some 30 years of diabetic model use, suggests poor model predictivity. Hence, while this model is currently regarded as the 'gold standard', its efficacy is debateable and will only likely be of relevance to diabetic foot ulcers, which comprise just $5 \%$ of all chronic wounds.

\section{Ageing models}

A deterioration in the efficiency of wound repair occurs in aged individuals (60); hence, aged mice can be used to model delayed repair within the elderly population (61). An alternative model of age-associated impaired healing is that of ovariectomy (OVX), where the surgical removal of the ovaries in female animals causes a loss of circulating hormones, mirroring the postmenopausal state (62). This model is used experimentally to mimic a number of human degenerative conditions such as osteoporosis (63), cardiovascular disease (64) and dementia (65). The OVX model shows comparable healing rates to aged mice, with the major advantage that mice do not require housing for 1-2 years before conducting the study. Additionally, the surgical procedure is not limited to a particular strain and can thus be exploited to examine delayed healing using the vast array of available transgenic mouse strains. This is particularly important as in wound healing studies, similar to so many other fields, phenotypes are invariably examined in young, not old, transgenic mice. We do note that

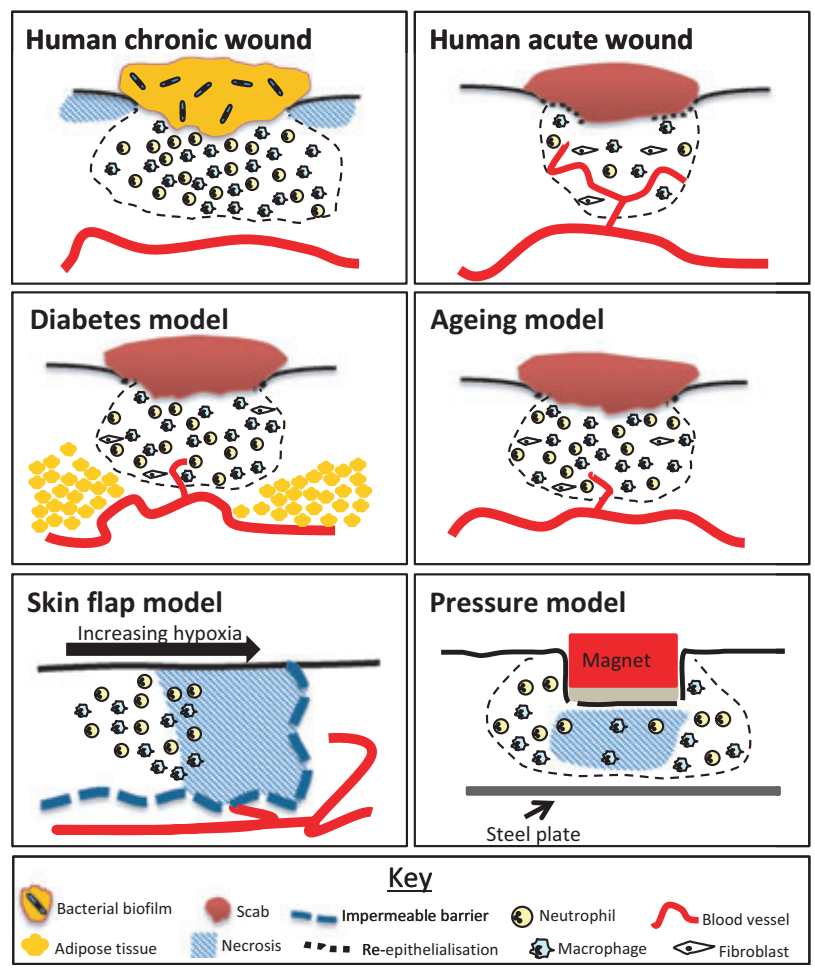

Figure 1. Impaired healing animal models attempt to recreate features of human chronic wounds. In humans, acute wounds heal through the coordinated processes of inflammation, proliferation and remodelling. Where this fails to occur, chronic wounds arise, typified by excessive inflammation and a retardation of subsequent phases of repair. Diabetic models attempt to recreate predisposing biological changes such as altered glycation or neuropathy. Similarly, aged and ovariectomised models mimic age-associated changes at both the local and systemic level, for example, elevated cellular senescence and excessive inflammation. Tissue hypoxia is a common underlying theme of poor healing in humans, which can be modelled using the skin flap model. Specific animal models employing magnets to compress tissue in vivo aim to mimic pressure sores in humans. 
transgenic models of premature ageing with healing defects are also available $(66,67)$. Interestingly, long-term glucocorticoid use in human patients in vivo, which causes skin changes similar to those during ageing, does not confer altered reparative potential in vitro (68). Ageing models possess an advantage over diabetic models in that they will be relevant to multiple chronic ulcer types (where age is a common risk factor) Fig. 1.

\section{Other models}

Chronic wounds are characterised by excessive inflammation, which inhibits subsequent phases of wound repair (69). Recent research has highlighted differences in wound bacterial colonisation as being important in the development of chronic wounds $(70,71)$, and this is now being modelled (72) but remains an emerging area. Researchers have attempted to recreate the effects of local tissue hypoxia in vivo using the skin flap model $(73,74)$. Here, a pedicle flap of skin is created with supplying blood vessels severed, creating an ischaemic gradient. The skin subsequently undergoes reproducible necrosis. Pressure ulcers can be modelled by placing magnets onto the skin (75), which can also be repeatedly removed and reapplied to mimic reperfusion of ischaemic tissue. An alternative model is available in pigs, which while being highly technical and time-consuming may provide the best reflection of pressure ulcers in humans (76). Burns can be modelled, with varying sizes and depths employed (77). Here, systemic effects occur and the mechanism differs to wounds made with a bladed implement (78).

For a comprehensive review of all available acute and pathological healing models and their methodologies, see (79). These models have been used predominantly to explore the processes involved during impaired repair. Each carries limitations for the evaluation of potential therapeutic compounds at the preclinical stage (see Table S2 online). Moreover, while available models display reduced overall rates of repair, a chronic wound is by definition one that entirely fails to heal. In this respect, no model currently reflects the human chronic wound environment.

The best is yet to come...

Clearly improved models are required, and researchers should be actively searching for 'refinement' where possible. A number of ethical considerations arise when exploring the development of an animal model which is entirely unable to heal wounds. Here, we hit on the crux of the matter in that the therapeutic amelioration of chronic wounds is being held back by a chicken egg scenario. The identification of beneficial therapeutic compounds is impossible without a suitable validated model, yet the development of more representative chronic wound models is hindered by the need to maintain animal welfare, but also by the lack of any positive controls required to validate the model. Until such time as improved models of chronic wounds become available, it is difficult to envisage radically improved therapeutics coming to market in the immediate future. In humans, chronic wounds are clearly multifactorial. Coupling together two or more existing delayed healing models in a combinatorial approach may confer an improved approximation of the human chronic state. This proposition could (and should) be tested experimentally.

\section{Conclusions}

Although in vitro assays have their limitations, they provide a useful high-throughput first step in drug development. More complex 3D 'organotypic' models hold great potential for in vitro wound assays but remain particularly underdeveloped. Studies using reproducible, highly validated in vivo models of acute wound repair have dramatically improved our understanding of the healing process. However, now that the normal healing continuum has been more fully elucidated, attention should focus on perturbed healing events. Though, the chronic wound models described here are far from ideal; they represent the best available at present. Given the current paucity of drug therapies for the amelioration of chronic or fibrotic wounds, the development of more robust models for evaluating new therapies for treatment of pathological healing is of utmost importance, while at the same time maintaining animal welfare.

\section{Acknowledgements}

DMA wrote the manuscript with full scientific input, critical reading and suggestions from $\mathrm{KAH}$ and $\mathrm{MJH}$.

\section{Conflict of interest}

$\mathrm{KAH}$ is an employee of Epistem Ltd.

\section{References}

1 Shaw T J, Martin P. J Cell Sci, 2009: 122: 3209 3213.

2 Werner S, Grose R. Physiol Rev, 2003: 83: 835870.

3 Russell W M S, Burch R L. The Principles of Humane experimental Technique. Methuen \& Co, London: 1959.

4 Bell E, Sher S, Hull B et al. J Invest Dermatol, 1983: 81: 2s-10s.

5 Kuchler S, Wolf N B, Heilmann S et al. J Biotechnol, 2010: 148: 24-30.

6 Kandyba E E, Hodgins M B, Martin P E. J Invest Dermatol, 2008: 128: 1039-1049.

7 Pistoor $\mathrm{F} H$, Rambukkana $\mathrm{A}$, Kroezen $\mathrm{M}$ et al. Am J Pathol, 1996: 149: 337-343.

8 Rijnkels J M, Whiteley L O, Beijersbergen van Henegouwen G M. Photochem Photobiol, 2001 73: $499-504$

9 Morita T, Tsuchiya A, Sugimoto M. Cell Tissue Res, 2011: 345: 379-390.

10 Bedoni M, Sforza C, Dolci C et al. J Dermatol Sci, 2007: 46: 139-142.

11 Portugal-Cohen $M$, Soroka $Y$, Frusic-Zlotkin $M$ et al. Exp Dermatol, 2011: 20: 749-755.

12 Doolin E J, Strande L F, Chen M K et al. J Burn Care Rehabil, 1999: 20: 374-376.
13 Xie $\mathrm{Y}$, Rizzi S C, Dawson $\mathrm{R}$ et al. Tissue Eng Part C Methods, 2010: 16: 1111-1123.

14 Vukelic S, Stojadinovic O, Pastar I et al. J Bio Chem, 2010: 285: 1980-1988. Epub 2009 Nov 10

15 Lindblad W J. J Biomater Sci Polym Ed, 2008: 19: 1087-1096

16 Klymiuk N, Bocker $W$, Schonitzer $V$ et al. FASEB J, 2012: 26: 1086-1099.

17 Grose R, Werner S. Mol Biotechnol, 2004: 28 147-166.

18 Bockamp E, Sprengel R, Eshkind $L$ et al. Regen Med, 2008: 3: 217-235

19 Fang R C, Mustoe T A. J Biomater Sci Polym Ed, 2008: 19: 989-1005.

20 White J A, Boffa M B, Jones B et al. Development, 1994: 120: 1861-1872.

21 Yoshii $Y$, Noda M, Matsuzaki $T$ et al. Dev Growth Differ, 2005: 47: 553-561.

22 Burd D A, Longaker M T, Adzick N S et al. Br Plast Surg, 1990: 43: 571-577.

23 Colwell A S, Krummel T M, Longaker $\mathrm{M} T$ et al. Plast Reconstr Surg, 2006: 117: 2292-2296.

24 Rowlatt U. Virchows Arch A Pathol Anat Histol, 1979: 381: 353-361.
25 Larson B J, Longaker M T, Lorenz H P. Plast Reconstr Surg, 2010: 126: 1172-1180.

26 Smith A R, Wolpert L. Nature, 1975: 257: 224 225

27 Rinkevich $\mathrm{Y}$, Lindau $\mathrm{P}$, Ueno $\mathrm{H}$ et al. Nature, 2011: 476: 409-413.

28 Beare $A H$, Metcalfe A D, Ferguson M W. J Anat, 2006: 209: 547-559.

29 Ferris D R, Satoh A, Mandefro B et al. Dev Growth Differ, 2010: 52: 715-724.

30 Scherer S S, Pietramaggiori G, Matthews J et al. Ann Surg, 2009: 250: 322-330.

31 Galiano R D. Michaels J t, Dobryansky $M$ et al. Wound Repair Regen, 2004: 12: 485-492.

32 Dyson M, Young S, Pendle C L et al. J Invest Dermatol, 1988: 91: 434-439.

33 Bitto A, Minutoli L, Galeano M R et al. Clin Sci (Lond), 2008: 114: 707-718.

34 Shah $M$, Revis D, Herrick $S$ et al. Am J Pathol, 1999: 154: 1115-1124.

35 Kloeters O, Tandara A, Mustoe T A. Wound Repair Regen, 2007: 15 (Suppl 1): S40-S45.

36 Wong V W, Sorkin M, Glotzbach J P et al. J Biomed Biotechnol, 2011: 2011: 969618.

37 Lucas T, Waisman A, Ranjan R et al. J Immunol, 2010: 184: 3964-3977. 
38 Campbell L, Emmerson E, Davies F et al. J Exp Med, 2010: 207: 1825-1833.

39 Posnett J, Franks P J. Nurs Times, 2008: 104: 44 45.

40 Sen C K, Gordillo G M, Roy S et al. Wound Repair Regen, 2009: 17: 763-771.

41 Keswani S G, Katz A B, Lim F Y et al. Wound Repair Regen, 2004: 12: 497-504.

42 Huang Z, Lu M, Zhu G et al. Wound Repair Regen, 2011: 19: 633-644.

43 Boulton A J. Diabetes Metab Res Rev, 2008: 24 (Suppl 1): S3-S6.

44 Boquist L, Hellman B, Lernmark A et al. J Cell Biol, 1974: 62: 77-89.

45 Baetens $D$, Stefan $Y$, Ravazzola $M$ et al. Diabetes, 1978: 27: 1-7.

46 Ring B D, Scully S, Davis C R et al. Endocrinology, 2000: 141: 446-449.

47 Tsuboi R, Rifkin D B. J Exp Med, 1990: 172: 245251

48 Fang R C, Kryger Z B, Buck D W et al. Wound Repair Regen, 2010: 18: 605-613.

49 Tkalcevic V I, Cuzic S, Parnham M J et al. Toxicol Pathol, 2009: 37: 183-192.

$50 \mathrm{Kim} \mathrm{J} \mathrm{H}$, Stewart T P, Soltani-Bejnood $M$ et al. J Endocrinol, 2006: 191: 437-446.

51 Allan M F, Eisen E J, Pomp D. Obes Res, 2004 12: $1397-1407$

52 Cho $Y R$, Kim H J, Park S Y et al. Am J Physio Endocrinol Metab, 2007: 293: E327-E336.

53 Gilbert E R, Fu Z, Liu D. Exp Diabetes Res, 2011 2011: 416254. epub 29th November 2011.

54 Michaels Jt, Churgin S S, Blechman K M et al. Wound Repair Regen, 2007: 15: 665-670.
55 Seitz O, Schurmann C, Hermes $\mathrm{N}$ et al. Exp Diabetes Res, 2010: 2010: 476969.

56 Greenhalgh D G, Sprugel K H, Murray M J et al. Am J Pathol, 1990: 136: 1235-1246.

57 Kitano $Y$, Yoshimura K, Uchida $G$ et al. Arch Dermatol Res, 2001: 293: 515-521.

58 Bevan D, Gherardi E, Fan T P et al. J Pathol., 2004: 203: 831-838.

59 Hardwicke J T, Hart J, Bell A et al. J Control Release., 2011: 152: 411-417. doi: 10.1016/j.jconrel.2011.03.016. Epub 2011 Mar 22.

60 Ashcroft G S, Greenwell-Wild T, Horan M A et al. Am J Pathol, 1999: 155: 1137-1146.

61 Ashcroft G S, Horan M A, Ferguson M W. J Anat, 1997: 190 ( Pt 3): 351-365.

62 Hardman $M$ J, Emmerson $E$, Campbell $L$ et al. Endocrinology, 2008: 149: 551-557.

63 Edwards M W, Bain S D, Bailey M C et al. Bone, 1992: 13: 29-34

64 Nikolic I, Liu D, Bell J A et al. J Mol Cell Cardiol, 2007: 42: 769-780

65 Dubal D B, Wise P M. Endocrinology, 2001: 142: $43-48$.

66 Massip L, Garand C, Paquet E R et al. FASEB J., 2010: 24: 158-172. Epub 2009 Sep 9.

67 Hiebert P R, Boivin W A, Abraham T et al. Exp Gerontol, 2011: 46: 489-499. Epub 2011 Feb 18.

68 Pratsinis $H$, Dimozi $A$, Pilichos $K$ et al. Exp Dermatol, 2011: 20: 529-531. doi: 10.1111/j.16000625.2011.01262.x. Epub 2011 Mar 16.

69 Loots M A, Lamme E N, Zeegelaar J et al. J Invest Dermatol, 1998: 111: 850-857.
70 Wolcott R D, Cox S B, Dowd S E. J Wound Care, 2010: 19: 272-278, 280-281

71 Edwards R, Harding K G. Curr Opin Infect Dis, 2004: 17: 91-96.

72 Schierle C F, De la Garza M, Mustoe T A et al. Wound Repair Regen, 2009: 17: 354-359.

73 Tatlidede S, McCormack M C, Eberlin K R et al. J Surg Res, 2009: 154: 112-117.

74 Minh T C, Ichioka S, Nakatsuka T et al. J Reconstr Microsurg, 2002: 18: 115-119.

75 Stadler I, Zhang R Y, Oskoui P et al. J Invest Surg, 2004: 17: 221-227.

76 Zhang J D, Mak A F, Huang L D. J Biomech Eng, 1997: 119: 406-408.

77 Greenhalgh D G. J Burn Care Rehabil, 2005: 26 293-305.

78 Posluszny J A Jr., Muthumalaiappan K, Kini A R et al. J Trauma, 2011: 71: 1288-1296.

79 Davidson J M. Arch Dermatol Res, 1998: 290 S1-S11.

\section{Supporting Information}

Additional Supporting Information may be found in the online version of this article:

Table S1. Selection of species for in vivo healing studies.

Table S2. Selection of an impaired healing model.

Please note: Wiley-Blackwell are not responsible for the content or functionality of any supporting materials supplied by the authors. Any queries (other than missing material) should be directed to the corresponding author for the article. 